Voix et Images

voixetimages

\title{
Cinématographie du Québécois : Voyageries à 24 images/seconde
}

\section{Gilles Thérien}

Volume 5, numéro 1, automne 1979

Jacques Godbout

URI : https://id.erudit.org/iderudit/200192ar

DOI : https://doi.org/10.7202/200192ar

Aller au sommaire du numéro

Éditeur(s)

Les Presses de l'Université du Québec

ISSN

0318-9201 (imprimé)

1705-933X (numérique)

Découvrir la revue

Citer cet article

Thérien, G. (1979). Cinématographie du Québécois : Voyageries à 24

images/seconde. Voix et Images, 5(1), 133-141. https://doi.org/10.7202/200192ar d'utilisation que vous pouvez consulter en ligne.

https://apropos.erudit.org/fr/usagers/politique-dutilisation/ 


\section{Cinématographie du Québécois: Voyageries à 24 images/seconde}

Tâcher de donner un sens à la compilation chronologique. Chercher dans les kilomètres de pellicule ce petit bout qui en dit plus que les autres. Défiler des cadres par milliers jusqu'à ce qu'un paysage, un geste, un visage nous retiennent. Tel est le défi que présente à l'analyse la production cinématographique des dernières années. La chronologie rassure mais elle est nettement insuffisante quand il s'agit d'accéder au sens des œuvres ${ }^{1}$. Nous nous laisserons aller à l'intuition en cherchant ici et là des regroupements, des correspondances, des contradictions partagées, des quêtes collectives.

Si, au début des années 60 , on peut saluer les rentrées littéraires de Bessette, de Renaud, de Marie-Claire Blais... etc, c'est sur un fond de recherche d'écriture bien de chez nous qu'on peut identifier leur démarche. Quand Gilles Groulx, Gilles Carle, Pierre Perrault et les autres prennent, à cette même époque, la caméra, ils devront forcer des écrans occupés par des œuvres étrangères qui ne manquent pas de qualité; ils tenteront de faire entendre leurs voix là où déjà la voix originale est disparue. Écrire sans être lu est un grand malheur, faire du cinéma qui n'est pas vu est un malheur encore plus grand: le cinéma n'est-il pas d'abord une industrie comme l'affirmait Malraux? Le spectacle rentable est-il conciliable avec la recherche de la parole? Voilà un peu les à-côtés de ce discours qui se donne comme objet le cinéma depuis les années 60 . Une quête de parole comme celle de l'écriture romanesque; un obstacle: les habitudes de spectacle dans lesquelles le public québécois se complaît volontiers.

Pour mieux saisir cette démarche, difficile tout le temps, parfois originale, il a semblé commode d'opérer des coupes dans le matériau cinématographique. Comme des décors successifs qui font progresser le drame, la géographie, l'archéologie et l'anthropologie viendront tour à tour occuper le fond de la scène. C'est en ce sens que cette "voyagerie" dans l'espace-temps tentera de reconstituer en mots plutôt qu'en images et sons, ce pays qui est la double visée du discours cinématographique, celle qui regarde dans l'objectif de la caméra et celle qui est regardée.

La géographie est d'abord la géographie physique, photographie des 
lieux, ce qui sert à les nommer mais aussi la géographie morale, celle qui divise ces mêmes lieux en bénis et maudits. L'espace est l'objet d'un choix physique et moral. II n'est jamais neutre, posé par hasard. L'archéologie reconstitue le passé á partir des vestiges. C'est une archéologie du regard. Elle cherche au delà de l'outil, le geste, celui qui, seul, est garant du temps, de la tradition, mais elle ne se contente pas de la reconstitution des actes, elle poursuit la reconstitution des sentiments que la pudeur, l'introversion ont ensevelis. L'anthropologie parle, elle, de ce curieux Homo québècensis insaisissable au point parfois de douter de son existence. Elle nomme la trace ou contemple le vide que l'on s'est habitué à appeler crise d'identité.

Ces trois points de vue ne sont pas gratuits. Ils correspondent à ce que l'école du cinéma québécois a enseignè même à ceux qui ont cru pouvoir s'en passer. Il existe bien une école, celle de l'Office national du film. Créé à des fins de propagande en $1939^{2}$, l'ONF devra attendre 1959 pour voir, après de virulents débats, la mise en place de l'équipe de production française, ce cadre administratif qui permettra aux cinéastes québécois de s'exprimer de plus en plus librement, moins soumis qu'ils sont aux aléas de l'économie. La tradition du documentaire constituera la base même du discours cinématographique et c'est à partir d'elle que s'élargira le désir de parole. Géographie, archéologie, anthropologie sont les articulations de la pratique de l'ONF et nous verrons qu'elles excèdent rapidement les cadres de l'"école".

Curieuse destinée d'un cinéma national appelé à décrire, malgré lui, le Québécois aux Canadiens et au reste du monde et ceci, au mépris des normes strictes de l'économie capitaliste qui sait faire avorter au nom de la loi du marché tout projet qui ne réflète pas l'idéologie dominante. Sur les deux plans, l'économique et le national, l'équipe française de l'ONF jouira d'une liberté frondeuse qui voudra se répercuter dans le marché habituel du film, de sorte qu'au seuil des années 80 , il est encore, toujours, question de créer un cinéma québécois rentable.

\section{Fondu au noir...}

Le paysage blanc, c'est celui de l'hiver. Sous la neige, se confondent villes et villages. L'hiver, c'est tout à la fois la misère et la pureté. La neige blanche, immaculée, est le linceul qui donne une apparence de dignité à ce qui, au fond, s'annonce comme corruption, tant celle de l'esprit que celle du corps.

C'est du corps perdu dans la neige qu'il est question dans Mon oncle Antoine, corps-cadavre perdu quelque part entre la pauvreté et l'alcoolisme. C'est l'esprit québécois aliéné par le capitalisme américain que recouvre pudiquement la neige qui tombe dans le dernier plan de $Q-b e c$ my love pendant que le discours-sermon du réalisateur-moralisateur prêche la conversion et la fin du cinéma. Dans Kamouraska ou dans Le Grand Rock, le sang répandu souille la neige. La violence vainc l'obstacle de l'hiver. Le mal a raison de cette saison bénie entre toutes, marquée du signe de l'intériorité. 
La neige a une valeur morale dont l'extension semble bien coïncider avec celle de l'espace du pays. Pourtant ce pays tout identique sous cet aspect devient singulièrement restreint au soleil. Les régions rurales sont grandement exploitées, des Laurentides à la Gaspésie, de l'Abitibi à l'ille-auxCoudres... Le pays rural prend nom: Thetford, Sainte-Adèle, la baie de James, Rouyn, Percé, le lac Saint-Jean... Québec, la ville, est plutôt absente. D'ailleurs, elle est la capitale de quoi ? Qui connaît ce tissu urbain crocheté au fonctionnaire, par millimètre carré ? Quel pouvoir s'exerce dans cette ville qui n'a de l'extérieur qu'un pouvoir d'attrait touristique. La meilleure utilisation cinématographique de Québec au plan de nos deux géographies, physique et morale, demeure celle de Hitchcock dans / confess. Et que dire de Montréal? Sauf en de rares exceptions, et certainement pas parmi les plus heureuses (Valérie, L'initiation, Bingo, Eclair au chocolat...), nous ne retrouvons pas l'ouest de la ville. Pour la majorité des cinéastes, la ville n'existe qu'à l'est et la géographie morale coïncide avec la géographie physique. Les films d'André Forcier sont certainement les plus représentatifs de cette caractéristique. L'ouest de la ville baigne pour le Québécois francophone dans la gadoue morale. Sous le signe de l'anglophone, de l'argent, de l'aliénation culturelle, le secteur ouest demeure un tabou visuel. Les cinéastes n'ont pas encore trouvé, sur pellicule, le chemin de Gomorrhe, même s'ils y habitent, ou refusent simplement de rendre compte de la petite (ou parfois grande) bourgeoisie québécoise dont ils sont membres.

A un niveau encore plus restreint, au plan spatial, c'est la maison qui devient le parangon de l'espace-moralité. Or, si la maison est, comme le veut Bachelard, à l'image de celui qui l'habite, il faut convenir qu'ici les escargots sont mal dans leur coquille. Mieux, on a souvent l'impression d'un bernardl'hermite. Ou bien, le logis reflète et ce, de façon caricaturale, une misère, une "kétennerie» disproportionnée, produit du phantasme-réalisateur, ou bien il se veut une illustration à peu près parfaite d'un décor québécois de style... la maison-musée. Il convient de dire ici que la représentation du décor ne permet pas toujours, et même pas souvent, au comédien de se sentir bien dans sa peau et de projeter l'image d'une vie en symbiose avec son habitat.

Parmi les films les plus représentatifs de ces choix, notons L'eau chaude l'eau frette, Patricia et Jean-Baptiste, Le chat dans le sac, Kamouraska, OK la liberté, La tête de Normande Saint-Onge.

Géographie physique, géographie morale, le pays est représenté, amputé de ce qui en fait la force vive, l'économie. Malicieusement, nous pourrions citer ici en guise d'exception Un homme et son péché. II est vrai que c'était avant les années 60 , c'est-à-dire avant la révolution tranquille. Le propos est aussi orienté presqu'exclusivement vers un milieu qui n'est pas celui des cinéastes de façon générale. Le manichéisme traditionnel de la littérature d'avant les années 60 (surtout) se trouve ici renforcé par une insistance sur les valeurs de la vie rurale opposées à celles de la vie urbaine; mentionnons pour exemples La piastre, La vraie nature de Bernadette, La dernière neige. Pendant que la littérature s'insinue dans la vie urbaine, dans 
ses mœurs, le cinéma québécois retourne à la campagne dans la foulée du retour aux valeurs de la nature du cinéma américain et du retour à la terre prôné par Duplessis. La campagne est un choix moral, et, en cela, reflète assez bien une longue tradition québécoise. Mais là où la littérature fait avancer les idées, le cinéma semble se contenter de cette représentation plus ou moins fidèle d'un monde qui correspond assez peu à ce que l'on pourrait appeler un projet collectif.

La prise en main du pays, la conquête des images de ce qu'il est, demeure encore très loin de la réalité qui anime rues et rangs. L'espace est quadrillé d'obstacles qui nous renvoient à notre histoire, à cette dimension "tamps" qui semble peser si lourd dans le discours cinématographique québécois. Non, "mon pays, ce n'est pas un pays", c'est une terre recouverte d'un voile qu'il faut bien, semble-t-il, tenter de soulever.

\section{Fondu enchaîné...}

Une partie importante des cinéastes québécois depuis 20 ans s'est employée à rechercher dans les vestiges de notre passé des images prégnantes de notre évolution actuelle. L'entreprise est très intéressante si on la compare à la naissance de tous les cinéastes nationaux qui ont connu leur période "épique». L'exemple d'Eisenstein est d'autant plus intéressant que, dans une certaine mesure, ses épopées cinématographiques se sont transformées en histoire. Il est normal que des cinéastes cherchent à cerner non seulement les lieux, le territoire mais aussi les événements reliés à la construction sociale sur ce même territoire.

II existe une archéologiẹ du regard dans le cinéma québécois. Réapprendre à voir, à se voir, alors que les films étrangers aux images exotiques inondent les écrans. La vie du marin, la vie de l'agriculteur, la vie de l'ouvrier nouvellement intégré au monde industriel sont autant de sujets qui vont constituer les principales mailles du réseau archéologique. St-Jérôme relate la naissance, la dynamique du syndicalisme. Le mépris n'aura qu'un temps et On est au coton poursuivront la même entreprise. L'ensemble de l'œuvre de Pierre Perrault nous conduit tout droit au cœur de l'archéologie du geste, la pêche au marsouin, la vie quotidienne, la construction des goélettes, la chasse aux caribous. Les images cherchent à reconstituer ce geste qui d'ancêtre à ancêtre est venu jusqu'à nous. Et, au-delà du geste, c'est aussi l'outil qui est repris, examiné de façon plus documentaire que fictive, l'outil à façonner le bois, à construire, à assurer la survivance. II faudrait ici ouvrir une parenthèse importante sur tout ce cinéma qui découvre et, en même temps, conserve les vestiges d'un passé révolu, annulé par le progrès technique. On pense ici à des cinéastes issus d'un même horizon idéologique que Perrault: Brault et ses films d'artisans; Gosselin, ses raquettes, son canot d'écorce. Le travail d'archivage est important. II s'étend maintenant au geste de la fête, à la musique issue du milieu, musique que l'on peut retracer sur tout le territoire de l'ancienne Nouvelle-France. 
Aux outils que la fresque documentaire ressuscite, aux comportements sociaux qu'elle rend si bien, il faut ajouter une autre archéologie qui tente désespérément de tirer l'histoire de l'épopée. Les films de Marcel Carrière sur Saint-Denis, la bataille de Châteauguay, les Zouaves, les film de Denis Héroux sur la bataille de Saint-Eustache, la conscription vue par Perron, les Ordres et Bingo, tous deux reliés à octobre 70 , voilà autant de tentatives de renouer avec la tradition épique. Mais les résultats sont dérisoires. L'outil est inefficace, un vieux fusil, un canon rouillé, quelques boulets, produits d'une coutellerie de fer-blanc. Des batailles qui sont gagnées par défaut comme à Saint-Denis, des guerres perdues, les seules vraies, des guerres qui ne font pas de morts comme l'envoi des Zouaves à Rome au $19^{e}$ siècle, une situation explosive qui n'arrive pas à être révolutionnaire, la névrose de 70 , voilà l'épopée famélique qui nous renvoie impitoyablement à ces plaines d'Abraham qui ont vu la fin d'un monde encore bien inconnu et la naissance d'un autre dont on perçoit très mal l'identité tératologique dans la multiplication de ses langues.

De l'agriculture à la culture, le fusil devient curieusement l'un des outils qu'on retrouve le plus au cinéma québécois. II tue pour la patrie, il tire pour défendre un territoire comme dans Les Smattes, il règle des comptes entre Blancs et Métis comme dans Red; puis il devient accessoire obligatoire du petit gangster, du voyou à dix cents quand il n'est pas l'organe " silencieux" d'un IXE-13. L'arme délaisse la nation pour le "pognon». Elle change de mains. Nous sommes loin de l'activité du chasseur. Mais il ne faut pas oublier que la ville est devenue le rendez-vous de tous les nomades, de tous ceux qui ont quitté la misère pour faire fortune... à n'importe quel prix. La fiction a moins bien réussi que le documentaire à reproduire un certain passé, parfois muet dans sa représentation mais si expressif.

De la même façon, on a tenté de faire une archéologie des sentiments. De la victoire, on a voulu tirer une fierté, une passion du territoire à défendre. De la défaite, on a extrait toute l'amertume et le désir de se reprendre un jour. Ce ne sont là que quelques sentiments qui viennent juger les reconstitutions historiques plus ou moins habiles. D'autres cinéastes ont voulu, eux, faire l'archéologie de leurs propres sentiments. C'est le cas du Chat dans le sac, de La terre à boire, de La vie heureuse de Léopold Z., de Taureau, de Réjeanne Padovani, de Pour le meilleur et pour le pire, des Dernières fiançailles, de J.A. Martin, photographe. Cette entreprise, souvent autobiographique, est particulièrement ardue surtout si elle est menée à une vitesse qui supprime toute possibilité de distanciation. C'est l'art d'avancer en regardant dans le rétroviseur. Les personnages n'ont que rarement l'épaisseur, l'étoffe qu'il faut pour laisser les sentiments naître chez eux, s'épanouir et s'exprimer à leur guise. Peut-être est-ce la trop forte contamination du documentaire qui fait qu'on a cru pouvoir "observer " les émotions « in vitro " sans reconnaittre chez elles les dynamismes, les illogismes normaux, à la fois prévisibles quant à leur occurrence et aléatoires quant à leurs formes.

L'archéologie des sentiments suppose une syntaxe de l'écriture cinématographique en pleine possession de ses moyens. Elle ne peut qu'accom- 
pagner un scénario dont la dramaturgie atteint la maturité. Il est alors normal que cette archéologie qui est nôtre n'en soit encore qu'aux premières strates, qu'à celles qui se nouent en surface mais dont les origines plongent profondément dans la dimension intérieure, celle qui réside au delà des masques, des caractères cinématographiques, là où le personnage peut esquisser un “Je suis" au lieu des innombrables et stériles "J'ai-J'ai pas "... Nous attendrons encore patiemment celui qui plongera dans l'âme québécoise à la recherche d'un "to be" fécond.

\section{Fondu enchainé...}

Ce n'est pas que manque une recherche de ce qu'est «l'homo québécensis "... Au contraire! Ici encore, parallèle étonnant, il existe un cinéma d'essai, si nous consentons à prendre ce mot dans son sens le plus littéraire.

Tous les films que nous avons nommés, ou presque, sans compter les autres, cherchent avec une minutie effarante à préciser les contours de cet homme québécois, à lui définir, quand cela est possible, une âme.

Anthropologie que l'œuvre des Perrault, Brault, Jutra, Perron, Dufaux, Lefebvre, Forcier, Carle, Lamothe, Godbout... Elle porte sur la dimension intérieure, sur la verticalité de l'homme actuel. Elle compare les jeunes entre eux, les vieux, les Français et les Québécois, les Américains et nous. Et c'est à l'intérieur de cette recherche anthropologique de différenciation que se cristallisent quelques grands thèmes qui définissent au moins dans notre imagination sinon dans notre imaginaire "l'homo québécensis". La mère hospitalière, rude, généreuse mais inflexible comme un père; le père silencieux, «maternalisé » dans son comportement de pourvoyeur; la pureté de la campagne opposée au vice urbain; la rigueur morale et sa contre-partie, l'anarchie; l'amour-fidélité et la fidélité sans amour, par principe.

II ne faut pas craindre de le dire: dans l'élaboration de son discours sur l'homme québécois, le cinéma est très en retard sur la littérature. Qu'on songe à Bessette, à Anne Hébert, à Langevin, à Marie-Claire Blais, à Aquin, à Jean-Jules Richard... etc. II semble évident que l'entreprise littéraire a réussi à mieux cerner les caractéristiques du milieu que le cinéma qui oscille entre des modèles européens ou américains. Peut-être le cas de Jacques Godbout est-il ici le plus symptomatique par sa double pratique et par l'indéniable supériorité de son écriture romanesque.

Ce qui pourrait sembler un paradoxe, montrer ce qu'est un Québécois, ne l'est peut-être pas autant que nous pouvons le croire. II suffirait de comprendre que l'image identifie moins que le mot. Elle se contente de représenter, d'illustrer. Elle manque du mordant que possède l'expression verbale qui peut véritablement se déployer selon toutes les dimensions. Les mots distillent des images floues qui se superposent les unes aux autres; l'image, elle, fige, désamorce le mot dans son pouvoir d'évoquer. C'est au niveau du comportement que l'image peut parler de façon précise. Or, existe-t-il un comportement typiquement québécois? 
Dès les premiers longs métrages, notamment dans $L \theta$ chat dans lo sac de Gilles Groulx, la quête de l'identité est posée tant sur le plan de la parole que sur le plan du comportement. Mais ce dernier ne parvient pas à traduire l'identité. Il expose seulement l'instabilité des personnages et des situations. L'identité devient la structure " creuse" de l'action dramatique. Cet état de fait persiste tout au long de la production cinématographique. Deux exceptions: les enfants et les vieillards. Melançon, Moreau, Dufaux, Perrault ont pu le constater : l'identité comme quête n'existe pas chez ceux qui sont trop jeunes pour l'avoir déjà entreprise et disparaît chez ceux dont la vieillesse a façonné le dernier masque. En ce sens, le regard anthropologique ne manque pas de justesse puisqu'il reproduit des comportements prévisibles dans une société normale. Le hiatus est créé dans la génération mitoyenne où la quête de l'identité s'accompagne généralement d'un manque d'identification au personnage. II arrive souvent dans le cinéma québécois que le comédien doive se substituer à son personnage, le nourrir comme il peut de ses phantasmes du moment en espérant satisfaire le voyeurisme de l'auteur. La quête de l'identité est double, dramatique au sens même de la dramaturgie, symbolique au plan du comportement.

Les films québécois fourmillent de cas de folie, de suicides réussis ou avortés, d'engluement dans l'alcool ou tout simplement dans l'ennui. La misère devient décrépitude. L'identité tend vers un point zéro où elle ne peut se remettre en question puisqu'elle n'existe pas assez pour cela.

A la décharge du cinéma québécois, il faut mentionner que la crise d'identité $a$, ici, contrairement à la littérature, des incidences très particulières sur les coûts de production et les recettes de diffusion d'un film. Alors que le coût moyen actuel d'un film se situe autour du million, il n'est pas étonnant de voir les cinéastes chercher à répondre à un modèle qui garantit les investissements. Récemment encore, un cinéaste, dont le cheminement n'est pas des plus heureux, annonçait bravement avoir réalisé un film “ international ». Une telle affirmation illustre bien ce que peut devenir la crise d'identité quand on veut, à tout prix, l'avoir déjà résolue. Le film « international »n'existe pas. Quoi de plus régional comme conception et comme facture que The last Picture Show de Bogdanovitch et pourtant son audience a été internationale parce que le niveau de son langage était universel. La littérature d'ici a mieux compris que le métier, l'espace du discours étaient les balises essentielles à toute universalité.

Sérieux de la crise ou crise du sérieux? Est-on pris à ce point par le problème de l'identité que l'humour n'aie plus sa place ? Le cinéma québécois a, presque systématiquement, occulté des thèmes particulièrement révélateurs. L'humour en est un, auquel on pourrait ajouter la superstition, la religion, la rouerie, le gros bon sens, une certaine forme de grivoiserie. Il faut le dire: peu en littérature, rarement au cinéma, voyons-nous éclater le carnavalesque, celui que chacun a connu au niveau comportemental, à un moment ou l'autre de son existence... de Québécois.

L'anthropologie entreprise ici a toute la rigueur et la froideur de 
l'argument de La chambre blanche de Jean-Pierre Lefebvre. La métaphysique passe par le congélateur de l'hiver et les gestes souffrent d'une trop haute altitude de questionnement. Tout devient bi-dimensionnel et souvent, simplification. L'identité québécoise repose, semble-t-il, sur un océan de tristesse, fait d'un passé mal digéré, d'un monolithisme intellectuel inquiétant.

\section{Fondu au nolr...}

On comprend mieux le dilemme dans lequel se débat le cinéma québécois. Issu d'une liberté qu'on lui a consentie mais qu'il n'a pas voulue autrement que comme un droit de s'affirmer, le cinéma québécois oscille continuellement entre les «manières», le régional, le national ou l'international, et les contenus. Les contraintes économiques se font de plus en plus sentir mais l'intervention continue de l'Etat ${ }^{3}$ permet de les rendre bien moins désastreuses qu'en d'autres pays. Or cette situation de relative facilité devrait favoriser une création libre de toute entrave. Ce n'est pas le cas. Les films ne sont pas vus et le nombrilisme va jusqu'à récupérer les fonds de tablette sous forme de festival.

La comparaison entre la littérature et le cinéma s'établit à deux niveaux. L'écrivain est seul à prendre ses responsabilités. II sait de plus en plus - ou de mieux en mieux - que le métier est histoire de temps. Ensuite, il fait d'abord face aux contraintes. Peu de romanciers peuvent se vanter de disposer des sommes dont jouissent un bon nombre de scénaristes. L'art, ici, naît de douleur! 4 Mais la page blanche est matrice. Les mots viendront s'y interféconder. La pellicule n'enregistre que ce que l'on prend la peine d'éclairer. Le cinéma qui n'a pas sa petite théorie de la lumière condamne la profondeur. Et c'est ici que le cinéaste québécois est le plus en retard. II ne fait pas, ou presque jamais, la théorie de son propre geste. II se fie, ou se confie, à ses atripes" sans en connaître toujours les humeurs.

La dramaturgie cinématographique, perdue dans l'image et le latent, oublie le discours. Le cinéma est mouvement du geste mais aussi de la phrase. Le problème réside non seulement dans le "comment dire" mais aussi dans le "ce qu'il y a à dire». L'écrivain comprend, ou on lui fait comprendre, qu'il faut avoir quelque chose à dire sous peine de ne pas être lu. Les cinéastes, eux, ont une méfiance exagérée de la littérature. Ils l'abordent avec circonspection comme s'il s'agissait d'un domaine réservé. Étrange et compréhensible qu'ils s'adonnent à l'adaptation des œuvres les moins adaptables ou encore, qu'ils n'ont pas perçu les mérites de la transposition de médium à médium.

Aujourd'hui encore, le seul cinéma d'ici qui ait la qualité de “l'endurance» est le cinéma d'inspiration documentaire. Quitter ce domaine, c'est vouloir maîtriser non seulement les paroles, mais encore le nationalisme 
qui érige les frontières et l'économie d'intervention qui élimine la compétition. Faire de la "fiction", c'est en connaître les règles et vouloir les assimiler pour pouvoir, un jour, s'en débarrasser dans un excès de liberté.

\section{Gilles THÉRIEN \\ Université du Québec à Montréal}

1. Il existe plusieurs compilations sur le marché. Signalons dans l'ordre de préférence. Pageau et Lever, Cinémas canadien ot québécois, Montréal 1977; les dossiers de la Cinémathèque québécoise; Houle et Julien, Dictionnaire du cinéma québécois. Fides 1978.

2. “art 9. L'OFFICE est établi pour entreprendre en premier lieu et favoriser la production et la distribution de films dans l'intérêt national, et notamment a) pour produire et distribuer des films destinés à faire connaître et comprendre le Canada aux Canadiens et aux autres nations " Loi de L'ONF 1950.

3. Par le truchement des organismes fédéraux comme l'ONF, la SDICC, société d'aide à l'industrie du long métrage, et d'un organisme provincial, l'Institut québécois du film.

4. André Gide.

Note: tous les films québécois cités dans le texte sont répertoriés dans les publications indiquées ci-haut. 